\title{
AUTOESTIMA E IDIOMA INGLÉS: UNA PRIMERA DISCUSIÓN
}

\author{
Raúl Montes de Oca Rodríguez \\ Profesor de las Escuelas de Lenguas Modernas y Formación Docente. \\ Universidad de Costa Rica.
}

Recibido 02-VII-2004 • Aceptado 10-VIII-2004

\begin{abstract}
Resumen: El artículo "Autoestima e idioma inglés: una primera discusión”, trata sobre la importancia que reviste la parte afectiva en el aprendizaje, en este caso, de una lengua extranjera como el inglés, en el medio costarricense. En ese sentido, se da una visión de lo que la ciencia cognoscitiva y las corrientes humanísticas proponen en cuanto a aprendizaje, rendimiento académico y aprendizaje de una lengua extranjera se refiere. Seguidamente, se hace un recorrido histórico del tema autoestima y se definen términos como autoestima, autoestima baja y alta. Además se analiza el aspecto adquisición/aprendizaje de un idioma y se ubica el tema en el sistema educativo costarricense. También se discute la situación de la enseñanza-aprendizaje de idiomas en nuestro medio y los aspectos que relacionan lo afectivo con el aprendizaje del inglés.
\end{abstract}

Palabras clave: Autoestima, conocimiento de sí mismo, educación cognitiva, educación afectiva, aprendizaje/adquisición de una lengua extranjera.

\begin{abstract}
Using Costa Rica's public educational context as a reference, this article analyzes the relationship between students' affective reactions and the learning of a foreign language as English. To meet this goal, both cognitive and humanistic trends are overviewed. Besides, the article examines proposals regarding learning, academic performance, and the learning of a foreign languages. This study includes brief historical background information on the topic of self-esteem. Other related terms are defined. The duality 'language acquisition' and 'language learning' is also analyzed from the Costa Rican educational system. This study also encourages further analysis and discussion of this controversial topic.
\end{abstract}

Key words: Self-esteem, foreign language acquisition.
El tema de la autoestima llama la atención porque, tal vez instintivamente, percibimos que el sentirnos a gusto con nosotros mismos influye en nuestra visión de mundo, es decir, en lo que sentimos, pensamos, decimos y hacemos, y porqué no decirlo, hasta en la dirección que tome nuestra vida. El amor a nosotros mismos, que no debe confundirse con egocentrismo, parece ser clave en el trabajo, el estudio, las relaciones interpersonales y la vida en general. Así, todo parece indicar que sin un autorrespeto, sin una autoimagen positiva, sin un autoconcepto firme, sin una autoconfianza, todos venidos de una sana autoestima, no hay forma de salir adelante. En criterio del autor de este artículo, ni el desarrollo de teorías tan creativas como la de las inteligencias múltiples, ni de "booms" tan impactantes como el de las estrategias de aprendizaje, compiten en el ámbito del estudio con el cambio positivo que puede realizar un educador en la vida de un estudiante, al ayudarle a sentir que él / ella es el mejor amigo (a) de sí mismo (a) y de que tiene la capacidad de salir adelante en sus estudios y en sus asuntos personales.

Ahora bien, sabido es que el tema de la autoestima no es nuevo. Buen número de investigaciones al respecto se han llevado a cabo, especialmente sobre la relación autoestima-rendimiento académico. Además, el tema pareciera que ha estado 
asociado a las corrientes de la psicología y la educación humanísticas (Ver Montes de Oca, 1995). Sin embargo, en estos tiempos, los teóricos de la información y las ciencias cognoscitivas han dado un paso adelante y nos presentan una propuesta, en cuanto a aprendizaje y rendimiento académico se refiere, basada en procesos mentales como la atención, la percepción, y la memoria (Williams; Burden, 1997, p. 15).

Asimismo, nos dicen que dicho enfoque es esencialmente mecánico y no tiene que ver con significados ni emociones y que el desarrollo de muchas de las así llamadas estrategias de aprendizaje, que entre otras cosas, incluyen estrategias para memorizar, ha sido desarrollado a partir de modelos de procesamiento de la información (Williams; Burden, 1997; p. 15).

Esta propuesta cognitiva dice conducir a un aprendizaje autónomo ayudando al estudiante a adquirir los conocimientos, las habilidades y las estrategias, que se necesitan para progresar, aprender más, resolver problemas y funcionar efectivamente en una cultura particular y en una sociedad cambiante. Este aprendizaje autónomo lo capacita para tomar control de su propio aprendizaje, con el objetivo fundamental de convertirlo en un pensador independiente y solucionador de problemas (Williams; Burden, 1997; p. 68).

Pero para ser pensadores independientes y solucionadores de problemas, no solo necesitamos del área cognoscitiva sino también de la afectiva. En ese sentido, Arnold y Brown (1999; p. 1) enfatizan que ni lo cognoscitivo ni lo afectivo tienen la última palabra, ni pueden separarse uno del otro. Por tanto, la cita de Williams y Burden deja escaso margen para la integración de un tema cono el de la autoestima, el cual está más relacionado con la vida emocional que con los aspectos cognitivos y los procesos mentales mencionados en los párrafos anteriores, pero el cual, al igual que el área cognitiva, es de fundamental importancia en el aprendizaje y para solucionar muchos de los problemas diarios.
Arnold (1999; p. XII) cita que aquellos educadores que no toman en cuenta el fenómeno de la autoestima, podrían perder algunos de los más esenciales ingredientes en el manejo de un aprendizaje exitoso.

Este artículo está, enfocado desde la perspectiva de un educador que ha investigado el tema de la autoestima y se ha preocupado por su relación con el rendimiento académico en general, y con el aprendizaje o adquisición del inglés como segundo idioma o lengua extranjera, en particular. Es por esto que parte de este trabajo se desprende de una tesis presentada en el año 1995 (ver Montes de Oca, 1995) en la Facultad de Educación de la Universidad de Costa Rica y sobre la cual se han venido construyendo algunas de las ideas aquí planteadas. Así pues, entre los objetivos del artículo se halla, primeramente, el plantear una discusión del tema aprendizaje/adquisición de una lengua extranjera como el inglés, dentro de un contexto amplio como lo es el de la autoestima, con el fin de elaborar elementos de juicio claros y objetivos que coadyuben al mejoramiento de la enseñanza de los idiomas en general y del inglés en particular, en las instituciones públicas y privadas de enseñanza. En segundo término, el artículo propone la clarificación de términos tan importantes como los de lengua extranjera y segunda lengua. En tercer lugar, se discuten las condiciones que deben cambiarse para llegar a un mejoramiento sustancial de la enseñanza-aprendizaje de un idioma. Finalmente, se analiza la relación autoestima-aprendizaje de un idioma y los factores que inciden en dicha relación. Se espera, así, contribuir a abrir un debate nacional sobre el tema del artículo.

\section{¿Adquisición o aprendizaje?}

En este punto cabe aclarar que algunos autores expresan que segundo idioma no se usa como opuesto de, o en contraste con, lengua extranjera, expresando que 
ya sea que la persona esté aprendiendo el idioma en forma natural, como resultado de vivir en una comunidad donde este se utiliza de manera oficial, o bien recibiendo instrucción en una aula, se acostumbra hablar genéricamente de adquisición de un segundo idioma (Ellis, 1997; p. 3). Sin embargo, para nuestro propósito seguiremos utilizando, en este artículo las expresiones inglés como lengua extranjera o adquisición-aprendizaje del inglés como lengua extranjera, puesto que en Costa Rica, nuestro idioma oficial es el español y no tenemos al inglés ni a ningún otro idioma como segunda (s) lengua (s), y pareciera, por tanto, más adecuado dar este tratamiento al tema. En síntesis, nos referiremos, pues, al acto enseñanza-aprendizaje del inglés y de qué manera se involucra la autoestima en dicho acto.

Así, al abarcar este tema nos veremos en la necesidad de clarificar quizás muy generalmente, conceptos tales como: autoestima, autoestima alta y baja, adquisición de una lengua extranjera y los aspectos afectivos involucrados en la adquisición del inglés.

\section{Un poco de historia}

Desde los remotos inicios de la civilización humana, la idea de autoimagen o autoconcepto ha sido reconocido como de trascendental importancia para el ser humano. Milenios antes de Cristo algunos movimientos filosófico - religiosos - los hindúes y budistas, proclamaban la importancia del autoconocimiento como un medio de desarrollo y evolución personal (La Rosa, 1991, p. 16, citado por Montes de Oca, 1995; p. 1). También los griegos acuñaron la frase "conócete a ti mismo" y finalmente, la frase bíblica "amar a tu prójimo como a ti mismo" vino a reiterar y resumir las ideas anteriores convirtiéndose en un mensaje para alcanzar la autorrealización personal.

Estas dos vertientes: "el conocimiento de sí mismo" y "el amor a sí mismo" han sido retomados una y otra vez por filósofos, psicólogos, educadores, místicos y guías espirituales de la humanidad en diferentes épocas y lugares. Es así como durante los años cuarenta, cincuenta y sesenta del siglo anterior, se produjeron movimientos que rescatan estos conceptos, en Oriente y en Occidente (Montes de Oca, 1995, p. 1). Así tenemos pensadores como Krishnamurti, quien en sus continuos viajes por Asia, Europa y America, acuñó frases como "solo el conocimiento de si mismo conduce hacia la paz", y "si no os conocéis a vosotros mismos, lo que pensáis y lo que sentís no puede ser verdadero" (Krishnamurti, 1981; p. 8), y también psicólogos y educadores como Carl Rogers y Maslow quienes desarrollaron ideas que colocaban a la persona como centro y motor de toda psicoterapia y educación. Rogers dio solidez científica a conceptos como que el ser humano tiene capacidad interna para alcanzar autocomprensión, modificar su autoconcepto, sus actitudes y su comportamiento, y de que basta con crear un clima de actitudes psicológicas facilitadoras para movilizar estos recursos (Montes de Oca, 1995; p. 2).

\footnotetext{
"Las personas a quienes se infunden dichas actitudes desarrollan una mayor autocomprensión, una mayor confianza en sí mismos, y una mayor habilidad para elegir su conducta. Aprenden de un modo más significativo y disponen de mayor libertad para ser y llegar a ser.

$\mathrm{El}$ individuo en este clima nutridor es libre para elegir cualquier dirección, pero en realidad selecciona los caminos positivos y constructivos. La tendencia de actualización es operativa en los seres humanos". (Rogers, 1989; p. 76-78).
}

Dichos temas: el autoconcepto y el autoconocimiento, fueron constantes durante los años sesenta del siglo pasado. Investigaciones y experimentos muy citados como el del "efecto Pygmalion" llevado a cabo en la universidad de Harvard, por el Dr. Rosenthal, demostraron que aquellos estudiantes de quienes se espera el éxito, con toda seguridad lo alcanzan; mientras que aquellos de quienes se espera el fracaso o a quienes se llena de comentarios negativos, presentan más dificultad para 
superar lo que hacen mal (Montes de Oca, 1995; p. 2).

Una autoestima baja o imagen negativa de sí mismo está asociada no solamente con un rendimiento académico bajo, sino también a conductas autodestructivas como el uso de drogas, el embarazo adolescente, el suicidio, la violencia en los colegios, y otras muchas. Una autoestima alta, por el contrario, conduce a la superación de las limitaciones (Montes de Oca, 1995; p. 3).

De esta manera, Rogers, Krishnamurti, Maslow y muchos otros han sugerido cambios en los sistemas educativos proponiendo que el alumno sea el centro del acto educativo donde su autoestima sea valorada en un clima de autoconfianza y de actitudes facilitadoras respetando los derechos del educando y promoviendo el autoconocimiento para que llegue a ser lo que la naturaleza haya dispuesto para él o ella y así ayudarlo (a) a prevenir las conductas autodestructivas mencionadas anteriormente.

\section{Definición de términos}

Pero bien, ¿qué entenderemos por autoestima en este artículo? En este artículo se entenderá autoestima como el juicio que efectúan los estudiantes de sí mismos. Este juicio está relacionado con sus actitudes, sentimientos y conocimientos de su apariencia física, aceptabilidad social y capacidad, los cuales son el resultado de dos aspectos:

- del aprendizaje y las experiencias obtenidas gracias a la interacción social y

- $\quad$ la influencia del entorno.

También, este juicio está sustentado por las valoraciones que se hacen de los estudiantes; por el factor étnico, su género y estado socio-económico. Este juicio no es estático, sino más bien evoluciona o cambia y se considera como la culminación de los estadíos, eslabones o etapas que se mencionan seguidamente (Montes de Oca, 1995; p. 53).

Al respecto, Novoa (1991) denominó escalera de autoestima a estos estadíos o eslabones por los que se supone atraviesa todo ser humano en la estructuración de una personalidad balanceada:

- Autoconocimiento,

- Autoconcepto,

- Autoevaluación,

- Autoaceptación,

- $\quad$ Autorespeto,

- Autoestima.

(Citado por Montes de Oca, 1995; p. 37).

\section{¿Autoestima baja y autoestima alta?}

Los individuos con autoestima baja presentan menos estabilidad emocional y confianza en sí mismos; por otro lado, presentan mayor grado de ansiedad, hipertensión, crisis, conflictos y menos tolerancia a la frustración. Son más tímidos, poco populares y su necesidad de aprobación es muy alta. Presentan alto temor al fracaso y a la muerte, gran tendencia a soñar despiertos y a posponer sus labores. También, son muy conformistas, sumisos, conservadores, dogmáticos y susceptibles de cambiar de opinión y de actitud (Novoa, 1991; Ortuño, 1978, citados por Montes de Oca, 1995; p. 45).

Aquellos cuya autoestima es alta, aprenden con más eficacia y eficiencia a controlar sus impulsos, y tienen una alta capacidad de retroalimentarse positivamente a sí mismos y retroalimentar a otros también. Su agresividad es baja y tienen una alta constancia para buscar soluciones positivas. No presentan problemas de identificación y son más positivos con 
su propio cuerpo y en cuanto al sexo en general. Por otra parte, buscan autorrealizarse, y ven la vejez con más optimismo. Al llegar a una edad avanzada se ven más integrados cognitiva y emocionalmente, y manifiestan un mayor grado de altruismo, así como también menor grado de recuerdos conflictivos (Novoa, 1991; Ortuño, 1978, citados por Montes de Oca, 1995; p. 45).

\section{¿Adquisición o aprendizaje de una lengua extranjera?}

Ahora bien, una vez clarificado lo anterior, se hace necesario enfocar el aspecto de la adquisición o aprendizaje de un segundo idioma. ¿Qué es adquisición y que es aprendizaje? Por muchísimos años educadores, políticos, analistas, padres de familia, estudiantes, y público en general nos hemos venido preguntando en Costa Rica como es posible que después de cinco años o más en enseñanza media los estudiantes de colegios públicos del país no sepan hablar inglés. Se lanzan diatribas al sistema educativo llamándolo defectuoso, obsoleto, anquilosado, y con otros tantos adjetivos peyorativos, también se califica la labor de los docentes del inglés.

¿Es cierto que nuestro personal docente y nuestro sistema educativo no funcionan con eficacia en términos de la enseñanza-aprendizaje del inglés, o es que hay otros tantos aspectos de fondo que se han venido soslayando?

Algunas investigaciones no tan recientes de la naturaleza de la adquisición de una lengua extranjera (Ellis, 1997), nos informan que la dificultad primaria para una comprensión a cabalidad de lo que sucede en la mente de un estudiante de una lengua extranjera debe iniciarse en la distinción o iluminación entre lo que es adquisición y de lo que es aprendizaje de un idioma. La adquisición de un idioma se refiere al desarrollo gradual de la habilidad de comunicarse naturalmente en un entorno en donde esa lengua sea dominante. El término aprendizaje se aplica al proceso consciente de acumular conocimientos de vocabulario y de la gramática de un idioma en un país donde este no es oficial. Así pues, una persona puede tener un buen conocimiento de las reglas gramaticales y de vocabulario del inglés, y sin embargo, ser incapaz de comunicarse oralmente. Es decir ha aprendido del idioma pero no lo ha adquirido. En ese sentido, tomando el caso de las matemáticas, podríamos decir que las matemáticas se aprenden no se adquieren (Yule, 1999, 191). De ahí la diferencia entre llegar a dominar un idioma y llegar a dominar el resto de los campos de estudio: los procesos cerebrales son diferentes.

Otro aspecto importante es el hecho de que aquellos jóvenes que se iniciaron en el estudio de una lengua extranjera después de los 12 o 13 años, como es el caso de más del noventa por ciento de los estudiantes costarricenses, tendrán una experiencia de aprendizaje a partir de la cual se supone no desarrollarían la habilidad de aquellos que tuvieron la posibilidad de estar inmersos en el idioma desde más temprana edad (Yule, 1999; p. 191). ¿Por qué?

Por lo que se conoce como "período crítico". Una vez que la pubertad ha pasado, cambios en la biología cerebral hacen difícil para cualquier persona adquirir otro idioma con plenitud. Así pues, se debe tomar en cuenta que hay un cambio en la dominancia hemisférica cerebral el cual produce una pérdida en la flexibilidad para adquirir un idioma (Yule, 1999; p. 191) (Schmitt, 2002; p. 116-117).

Sin embargo, otras investigaciones nos dicen que el período óptimo para la adquisición de una lengua extranjera se haya entre los 10 y los 16 años, cuando la flexibilidad de la facultad de adquirir un idioma aun no se ha perdido del todo (Yule, 1999 ; p. 192). Lo anterior se cumple siempre y cuando se haya dado una inmersión en el idioma lo suficientemente larga e intensa como para que el proceso de adquisición se cumpla. En nuestro país, en la actualidad, pareciera que la enseñanza del inglés en una institución pública de ense- 
ñanza general básica y/o diversificada está más bien orientado hacia el conocimiento y aprendizaje de esa lengua extranjera, ya que lo asociado a la adquisición se produce más que todo en una institución privada de enseñanza bilingüe que cubre etapas que van desde prescolar hasta el bachillerato en enseñanza media, donde el estudiante se inicia en el conocimiento de una lengua extranjera desde períodos previos a la pubertad, inmerso en un ambiente bilingüe y con acceso a otros aspectos relevantes que van desde intercambios con países de habla inglesa hasta importantes recursos multimedia.

\section{Autoestima y adquisición de una lengua extranjera}

Ahora bien, si esto es así, ¿que papel juega la autoestima en el aprendizaje de una lengua extranjera? Desde Krashen (1982) hasta Gardner (2001) se ha escuchado, con la misma fuerza, la otra cara de la moneda en la adquisición de una lengua extranjera: la importancia de los factores afectivos o emocionales que influyen en el aprendizaje. Krashen nos informa de la hipótesis llamada "filtro-afectiva", la cual afirma que la condición para un aprendizaje exitoso se da cuando el alumno está motivado a aprender el idioma extranjero y está receptivo a la información que se le dé (Schmitt, 2002, p. 118).

Gardner (2001, p. 53), por otro lado, nos amplía aún más la noción de aprendizaje y su relación con la parte emocional o afectiva cuando afirma:

\footnotetext{
"En mi discusión inicial de la inteligencia intrapersonal, también destaqué sus orígenes en la vida emocional y su fuerte vínculo con factores afectivos. Sigo pensando que la vida emocional es un ingrediente fundamental de la inteligencia intrapersonal, pero ahora destaco más el papel esencial que desempeña esta inteligencia en las decisiones que toma una persona a lo largo de su vida. Además, hoy creo que es necesario tener en cuenta las facetas emocionales de cada inteligencia en lugar de limitar las emociones a una o dos inteligencias personales."
}

Lo anterior arroja un poco de luz sobre la discusión del por qué un estudiante de enseñanza media de un colegio público no se comunica eficientemente en inglés luego de cinco años o más de estudio: por un lado, aspectos biológicos; por otro, aspectos relacionados con la parte afectiva. Veamos como lo afectivo juega un papel fundamental, pero antes analicemos algunos otros aspectos que limitan el aprendizaje.

\section{Un poco de todo}

Solamente por un instante imaginemos el cerebro de un adolescente cualquiera atravesando el mare magnum de la pubertad:

- Un proceso intenso de cambio psicobiológico.

- Una gama multifacética de intereses.

- Una variedad de sensaciones y emociones.

- Un bombardeo de información audiovisual y multicultural.

A lo anterior agreguemos

- Una dosis de problemas en el hogar que nunca faltan:

- Drogas.

- Violencia doméstica.

- Hogares disfuncionales.

- Padres o madres alcohólicos.

- Problemas económicos.

- Problemas de nutrición y de salud.

- Una dosis de crisis existencial.

$\mathrm{Y}$, finalmente, como resultado de todo lo anterior, un bajo autoconcepto que lleva hacia una baja autoestima, y tendremos un cuadro, que aunque muchos expertos en psicología tachen de negativo, dramático, muy generalizado o hasta exagerado, es puntos más o puntos menos, algo con lo cual, los docentes de escuelas y colegios públicos, y 
por qué no decirlo también privados, nos hemos visto enfrentados durante años y ante los cuales poco o nada hemos podido hacer por más frustración y consternación que todo el asunto nos haya causado.

¡Por supuesto que no se puede generalizar!, pero gran cantidad de adolescentes atraviesan dichas circunstancias (u otras peores) y todo eso mina su capacidad de aprendizaje y con mayor razón su capacidad de adquisición de una lengua extranjera lo cual de por sí, a partir de los 12 o 13 años, pareciera comenzar a hacerse más complicada, como, se vio, por asuntos meramente biológicos.

\section{Autoestima y adquisición/aprendizaje del inglés como lengua extranjera: ¿hacia dónde podemos avanzar?; o ¿hasta dónde podemos llegar?}

Cantero (2004; p. 4 ) cita una medición llevada a cabo por la psicóloga Luz Valdez con 349 niños de 9 a14 años de un centro educativo público de San José. En ella se encontró que más de la mitad de esa población está por debajo del promedio de una inteligencia emocional aceptable". Por su parte, Arnold (1999, p. XIII) cita a Menuhim quien señala que la educación de hoy en día está dirigida hacia el entrenamiento de habilidades cognitivas más que al de las emocionales de los alumnos. Lo anteriormente expuesto es básicamente cierto ya que como se expresó párrafos atrás, hoy en día, las ciencias cognoscitivas nos hablan sin más, de procesos mentales, entrenamiento en estrategias de aprendizaje y aprender a aprender. En este contexto, dejando a un lado el desarrollo de la inteligencia emocional, lo que se verá en el siguiente apartado como referencia en la relación autoestima-adquisición de una lengua extranjera, aunque enmarcado por autores de una cultura como la anglosajona, no deja de ser menos cierto o importante -pues es claro que todos los seres humanos tenemos el mismo equipo afectivo, desde Costa Rica hasta Escocia y Katmandú.

Sin embargo, antes de proceder, se hace imperativo hacer un alto en el camino y plantearse dos preguntas claves en el proceso de adquisición/aprendizaje de una lengua extranjera en nuestro medio: ¿hacia dónde podemos avanzar?, y ¿hasta dónde podemos llegar? Es sumamente importante plantearnos de una vez por todas si en nuestro medio, los estudiantes de enseñanza media pueden avanzar hacia la adquisición de una lengua extranjera como el inglés o, si más bien debemos tener claro que nuestra meta como docentes de una lengua extranjera debe llegar hasta el punto en que nuestros estudiantes alcancen algún aprendizaje de esa lengua, y así, por un lado, dejar de atormentarnos preguntándonos ingenuamente el por qué nuestros alumnos, luego de cinco años de secundaria aún no hablan inglés, por un lado. Por otro lado, si tenemos claro lo anterior, habremos dejado de autoengañarnos pensando que dicha meta será posible.

La tesis, o criterio que se sostiene en este artículo basada en hechos como los que se mencionaron anteriormente, es que mientras no se cambien las condiciones de enseñanza de los idiomas en Costa Rica, el único camino que tenemos es de dirigir a nuestros estudiantes a un aprendizaje más o menos superficial del inglés como lengua extranjera.

\section{Condiciones que deben cambiarse}

Pero, ¿cuáles son esas condiciones que deben cambiarse para que nos enrumbemos hacia la adquisición y no al aprendizaje del inglés como lengua extranjera? Hay muchas. La mayoría son ampliamente conocidas. Algunas son:

- Mayor inmersión del alumno en el inglés. Diversas investigaciones (Yule, 1999, p. 190) nos dicen que algunas razones obvias para los problemas que se experimentan en la 
adquisición de un segundo idioma se deben al hecho de que la mayoría de la gente trata de aprender un idioma estudiando unas pocas horas por semana, junto a otras ocupaciones, y con un primer idioma que utilizan para la mayoría de las comunicaciones diarias. Eso implica que para mejorar sustancialmente el nivel de inglés en nuestra población estudiantil, necesariamente se requiere un mayor número de lecciones por semana: con tres o cinco lecciones resulta muy mediocre lo que se puede alcanzar y no es culpa del docente ni del estudiante que este no pueda hablar el idioma luego de cinco o más años en secundaria. Golcher (2004; p. 8 A) dio a conocer a la opinión pública el hecho de que de los cuatrocientos cincuenta y tres colegios públicos, "solo once ofrecen una verdadera educación bilingüe”. Además expresa que la mayoría de los estudiantes reciben en promedio cinco lecciones semanales, mientras que en dichos once colegios, los alumnos reciben el doble de clases, aparte de Estudios Sociales y Ciencias en una lengua extranjera como el inglés. Asimismo, Golcher cita al viceministro de Educación, Wilfrido Blanco, haciéndonos conscientes de que, "en la mayoría se da un inglés gramatical y técnico, pero no salen con un verdadero dominio de la lengua sino con las bases necesarias para seguir estudiando".

Golcher (2004; p. 8 A) también expresa que esa puede ser la razón por la cual los estudiantes de noveno y undécimo fallen en comprensión, expresión, y descripción en las pruebas nacionales ya que el inglés es considerado una "materia básica y no complementaria", sin embargo, la cantidad de lecciones de inglés en comparación con Estudios Sociales, Ciencias, Español y Matemáticas es menor: "mientras en Español se dan unas ocho horas semanales, de inglés sólo se dan tres aproximadamente".

- Grupos de no más de 10 o 15 estudiantes -aunque el autor carece de evidencia fundamentada en investigación, su experiencia de más de veinte años enseñando inglés tanto en primaria, en enseñanza diversificada y a nivel de instituciones comerciales y universitarias, aunado al sentido común, indica que, utópico como suena, este es uno de los pilares básicos en la adquisición de un idioma-; con grupos de 35 o más alumnos la labor de un profesor de inglés u cualquier otro idioma es casi imposible. Una alternativa sería que los grupos tuvieran dos profesores...! sin comentarios!

- Mayor capacitación docente:

A. Las universidades Públicas y privadas del país deberían plantearse la urgencia de dar una preparación académica más sólida a quienes se dediquen a la enseñanza de idiomas tanto en primaria como en secundaria. Al respecto, Golcher (2004; p. 8 A) comenta el caso del programa de Lenguas Extranjeras creado en 1994. Según se cita "solo en un poco más de la mitad de las tres mil seiscientas setenta y nueve escuelas se da inglés." A lo anterior hay que abonarle el hecho que se consigna en este apartado ya que el viceministro afirma que no hay educadores preparados para cubrir ese frente.

B. También deben preocuparse por crear en los educadores la conciencia de que los alumnos necesitan un estímulo importante en su 
condición socio-afectiva y hacia este punto es que dirigiremos el resto del artículo.

El problema fundamental es que para que todas las condiciones aquí mencionadas se hagan realidad se necesita de decisiones políticas que van más allá de cualquier esfuerzo docente. Además, se necesita de un pensamiento claro basado en información de las ciencias del lenguaje. Sin embargo, la condición del punto 3B del apartado anterior sí está al alcance de cualquier docente ya que tiene que ver con áreas como las relaciones interpersonales, la estimulación de la autoestima y otras que se esquematizan a continuación.

\section{Autoestima y aprendizaje del inglés}

Oxford (1999; p. 62) cita que quienes han tenido poco o ningún éxito en el aprendizaje de un idioma con frecuencia tienen una autoestima más baja que quienes han tenido éxito. Esto por cuanto el aprendizaje de un idioma priva al individuo de su medio normal de comunicación, de su libertad para cometer errores y su habilidad para conducirse como gente normal. En ese sentido, una persona cuya autoestima general tiende a ser normal, puede sentirse amenazada o disminuída y su autoestima particular (en lo relativo al aprendizaje de un idioma) deteriorada si para ella, por alguna razón, el aprendizaje del inglés tiene un más alto nivel de dificultad que para el resto de sus compañeros.

Andrés (1999; p. 87) a su vez considera que el sentimiento de incompetencia proviene de una autoimagen deteriorada, un miedo al fracaso (citando al psicólogo Nathaniel Branden quien considera estos casos como de una baja autoestima). Además, Andrés (1999, p. 87) expresa que hay una fuerte correlación entre la autoestima, las relaciones sociales y el rendimiento académico, lo cual es algo que puede atestiguarse todos los días en un aula.
Andrés (1999; p. 88) también cita otros autores quienes mencionan que la autoestima conlleva 5 componentes:

- $\quad$ un sentido de seguridad,

- $\quad$ un sentido de identidad,

- un sentido de pertenencia,

- un sentido de propósito y

- un sentido de competencia personal.

Andrés (1999; p. 89) asimismo enfatiza que muchos estudiantes, consciente o inconscientemente, son humillados en las aulas en persecusión del mítico "high standard" o alto desempeño. Pero esto no solo se ve en educación general básica o diversificada sino también a nivel universitario, si hemos de creer en algunas de las evaluaciones que hacen de ciertos profesores los mismos estudiantes de la Universidad de Costa Rica. Pero esto es un asunto para un futuro artículo. Continuando con lo anterior, Andrés (1999; p. 89) nos recuerda que una aula debe ser en realidad el lugar donde las debilidades de los estudiantes se revelen como un espacio para el crecimiento y el desarrollo.

En otro orden de cosas, Andrés (p. 99) manifiesta una verdad que debe ponernos en guardia contra una explosión de premios y medallas para incrementar de forma positiva la autoestima de los alumnos y es que los programas y esfuerzos para hacer que los estudiantes se sientan bien tiene pocos efectos duraderos ya que por lo general fallan en fortalecer las fuentes internas de la autoestima relacionadas con la integridad, la responsabilidad y el logro, enfatizando que solo dirigiéndose a estas áreas es que se puede construir efectivamente la autoestima.

¿Qué necesitaríamos desarrollar? Amor y apoyo a otros, aprendizaje profundo de sí mismos, dar espacio para que 
los demás también crezcan y un lenguaje enriquecido para expresar sentimientos y comunicar ideas (Andrés, 1999; p. 99).

Arnold y Brown (1999; p. 3) resumen claramente lo que el factor afectivo en el aprendizaje y enseñanza del inglés representa:

Cuando se puntualizan las ventajas de enseñar habilidades de razonamiento en una clase de idiomas, Chamot enfatiza la importancia del aprendizaje cooperativo. La autora nota que el trabajo cooperativo en el aprendizaje de idiomas ayuda a desarrollar la así llamada inteligencia interpersonal, tal y como Gardner la definió. Esta se caracteriza por la habilidad de entender y responder efectivamente a otros (1995:4). Este es un paso en la dirección del conocimiento emocional.

Por otra parte, Arnold y Brown (1999; p. 4) mencionan que Freudenstein ha sostenido que en nuestro mundo en continua agresividad, la enseñanza de la paz tiene un rol vital en las clases de idiomas como el inglés. Los autores también mencionan otra diversificación de objetivos que dan una perspectiva diferente a lo que ha sido el rol tradicional del docente del inglés:

- la importancia de distinguir claramente entre el ser y el hacer, es decir que un docente debe saber y hacer las cosas en clase correctamente,

- $\quad$ pero también debe ser un individuo positivo, moralmente incuestionable,

- además, con una gran claridad de quien es como individuo.

- Lo anterior significa que todo docente debe poseer una alta inteligencia emocional lo que hace la diferencia en el proceso de aprendizaje de un idioma, desde el punto de vista del alumno.

Arnold y Brown (1999; p. 7) también expresan que reconocidos especialistas han apoyado un enfoque integrado en la enseñanza de los idiomas, comentando que las teorías cognitivas del aprendizaje puras serán rechazadas a menos que se le asigne un rol a la parte afectiva.

\section{Otros aspectos que relacionan lo afectivo con el aprendizaje de una lengua extranjera}

\section{Factores individuales}

Según Arnold y Brown (1999; p. 8) el proceso de adquisición de una lengua extranjera o segundo idioma, está fuertemente influido por rasgos individuales de la personalidad del estudiante. Un ejemplo de esto es la ansiedad. Este es uno de los factores que más impiden el aprendizaje y está relacionado con frustración, duda, aprensión y stress: el hecho de ser ridiculizados por dar una respuesta equivocada en clase, o bien por anteriores experiencias negativas que se han ido acumulando.

Como se dijo anteriormente, el aprendizaje de una lengua extranjera deja a la persona vulnerable frente a otros. El advenimiento de los métodos comunicativos que enfatizan el expresar oralmente aspectos personales, tales como sentimientos, puede, si no se hace con habilidad, provocar situaciones de ansiedad que pueden llevar a más frustración y desconcierto. Detengámonos solo por un momento a considerar un escenario en donde a un estudiante con adecuación curricular significativa, quien tiene problemas de timidez y tartamudeo, se ve obligado a pasar al frente de sus compañeros a dar el famoso "speech" a través del cual "hablará" de algo que posiblemente no le gusta, o que ni siguiera sabe de que se trata, pues lo memorizó mecánicamente la noche anterior; en un lenguaje de extraña pronunciación, que posiblemente detesta; agreguemos al escenario unos cuantos compañeros burlistas y crueles, y sentiremos como un profesor puede asestar un rudo golpe a la autoestima y agregar más ansiedad a un alumno y a provocar situaciones que después se habrán de lamentar. 


\section{La inhibición}

La crítica sarcástica y las palabras que ridiculizan debilitan grandemente el ego. $\mathrm{Al}$ aprender una lengua extranjera se debe tratar de ser capaz de tomar riesgos y equivocarse.

Los niños y jóvenes desarrollan inhibiciones cuando toman mayor conciencia de la necesidad de proteger un ego frágil, evitando lo que puede amenazarlo (Arnold y Jane; 1999; p. 10).

A esto hay que agregar la importancia a nivel afectivo que tiene el docente para el estudiante. En los años 70 y 80, investigaciones sobre el efecto que producía el alcohol o el valium en la pronunciación de los estudiantes resultaron poco concluyentes; sin embargo, demostraron que la persona que administraba el test, producía un efecto más beneficioso en la ansiedad de los participantes que el mismo alcohol o valium. Los resultados apuntan a la hipótesis de que los factores humanos más que las sustancias químicas pueden ser beneficiosos en la reducción de la ansiedad (Arnold y Jane, 1999; p. 10).

\section{La extroversión/introversión}

No hay prueba investigativa concluyente de si el factor extroversión-introversión está directamente relacionado con el éxito en las clases de inglés, pero si se puede afirmar que ciertas actividades son más apropiadas para uno o para otro (Arnold y Jane, 1999; p. 11).

\section{La autoestima}

Canfield y Wells (1994) sugieren que el aspecto más importante que un docente puede tomar en cuenta para ayudar a sus estudiantes emocional e intelectualmente, es el crear un ambiente de apoyo mutuo, siendo esencial la seguridad y la ayuda en el aula. Además tienen que reconocer que son valiosos y que recibirán afecto y una mano amiga (Arnold y Jane, 1999; p. 12).

\section{Conclusiones}

Lacorrelación autoestima-rendimiento académico-aprendizaje de una lengua extranjera es apasionante y llena de aristas que no se pueden cubrir en un corto artículo como el presente; sin embargo, es importante enfatizar los siguientes aspectos que pueden servir de puntos de apoyo para futuras investigaciones:

- La autoestima, el autoconcepto y la autoimagen son constructos que están interconectados, interactúan $\mathrm{y}$ se interrelacionan continua $\mathrm{y}$ constantemente.

- Algunas investigaciones parecen orientar hacia el hecho de que los estudiantes de enseñanza media tienden a no conocerse, no aceptarse y no apreciarse de una manera sana, sin distingos para hombres o mujeres (Montes de Oca, 1995; p. 137).

- Las evidencias sugieren que existe una correlación significativa entre autoestima y rendimiento académico.

- La investigación hace pensar que hay una correlación significativa entre autoestima baja y la actitud negativa hacia el aprendizaje de una lengua extranjera o entre autoestima baja y el sentimiento de incompetencia para el aprendizaje de esa lengua.

- Se puede hablar simultáneamente de autoestima baja en relación con un campo específico de estudio, pero a la vez se puede hablar de alta autoestima en otro. Por ejemplo, un estudiante puede tener una baja autoestima en el aprendizaje del inglés pero alta en el de matemática.

- Parece haber relación significativa entre el desarrollo de una alta autoestima en general y el mejoramiento del 
rendimiento académico general; por otra parte podría haber una relación significativa entre el desarrollo de una autoestima alta específicamente relacionada con una materia como el inglés y el mejoramiento en el rendimiento académico de la misma.

- En la adolescencia, la presión del grupo determina la autoestima (González, 1998, P. 58).

- $\quad$ Actividades en clase que conduzcan a un desempeño exitoso ayudan a mejorar la autoestima en esa materia.

- La autoestima del docente y del personal administrativo juegan un papel transcendental en el desarrollo de la autoestima específica y general. Docentes y administrativos deben estar conscientes de su propia autoestima: no se puede dar ni hablar de lo que carecemos (Montes de Oca, 1995; p. 6).

- El conocimiento de sí mismo al identificar qué le gusta y qué no de la vida, la aceptación de esos aspectos, y la definición clara de lo que queremos son elementos que pueden ser tratados en clase.

- Recordemos que el ambiente del cual proviene un estudiante ha influido en su baja autoestima. Hay un trasfondo del que los docentes no somos conscientes y el cual determina lo que el estudiante trae al aula.

- En relación con la autoestima, es importante ayudar al estudiante a ser él mismo: para que poco a poco se convierta en lo que la naturaleza ha diseñado para él o ella. En este sentido, podríamos utilizar como punto de partida filosófico el siguiente escrito de Carl Rogers:

"Así pues, al facilitar un clima psicológico para permitir que las personas sean -tanto si se trata de clientes, estudiantes, obreros, como miembros de un equipo- no nos ponemos en manos del azar. Nos infiltramos en una tendencia que impregna la totalidad de la vida orgánica, que permite alcanzar la máxima complejidad de la que el organismo sea capaz. Y a una escala todavía mayor, creo que sintonizamos con una potente tendencia creativa que ha formado nuestro universo, desde el diminuto copo de nieve hasta la mayor de las galaxias, desde la ameba hasta el más sensible y dotado de los seres humanos. Además, puede que estemos tocando el borde mismo de nuestra capacidad para trascender nosotros mismos, para crear direcciones nuevas y más espirituales en la evolución humana.” (Rogers, 1989; p. 78).

El párrafo anterior da pie para enfatizar la importancia de los temas tratados en este artículo. En este sentido, se quisiera insistir en el papel que juega lo que Rogers llama la totalidad de la vida orgánica y lo implican estas palabras en el proceso enseñanza-aprendizaje de una lengua extranjera. Es decir, tomar la totalidad de los aspectos cognitivos y la totalidad de los aspectos afectivos y verlos como un solo aspecto con dos caras que no se pueden ignorar. Asimismo, es impostergable el tomar conciencia de los eventos psicobiológicos que inhiben el aprendizaje adolescente y revisar el tema de la autoestima, poniéndolo sobre el tapete de la capacitación docente. Siguiendo esta línea de pensamiento, debemos insistir, también, en las condiciones que deben cambiarse para fortalecer la enseñanza-aprendizaje del inglés. Entre ellas están el aumentar el número de lecciones semanales y una mayor capacitación docente no solo en áreas lingüísticas y metodológicas, sino también en áreas donde el factor afectivo pueda ser estudiado con mayor profundidad: todo docente del inglés debe estar enterado de la relación autoestima-rendimiento académico y manejar, por lo menos, 
información básica tomada de investigaciones nacionales o internacionales.

\section{Referencias bibliográficas}

Arnold, Jane. Affect in Language Learning. United Kingdom: Oxford University Press, 1999.

Cantero, Marcela. "Emocionalmente inteligentes". La Nación. P. 4 Viva; 14 abril de 2004 .

Ellis, Rod. Second Language Acquisition. Hong Kong: Oxford University Press. 1997.

Gardner, Howard. La Inteligencia Reformulada. España: Editorial Paidós. 2001.

González, José Francisco. La Autoestima. España: Edimat Libros, 1998.

Gólcher Raquel. "Educación bilingüe llega a muy pocos estudiantes". La Nación: p. 8 A, 14 de abril de 2004 .

Krashen, Stephen. Second Language Acquisition and Second Language
Learning. Great Britain: Pergamon Press Ltd., 1984.

Krishnamurti, Jiddu. Solo el Recto Pensar Conduce a la Paz. México, Editorial Orion, 1981.

Montes de Oca, R. Raúl. Desarrollo de la Autoestima del Estudiante de Educación Diversificada, desde la Perspectiva de la Educación Humanística. San Pedro: UCR, tésis, 1995.

Oxford, Rebecca. Language Learning Strategies. Boston: Heinle and Heinle Publishers, 1990.

Rogers, Carl. El Camino del Ser. Argentina: Editorial Troquel, 1989.

Schmitt, N. An Introduction to Applied Linguistics. United States: Oxford University Press, 2002.

Williams Marion, Burdern Robert. Psychology for Language Teachers. United Kingdom: Cambridge University Press, 1997.

Yule, George. The Study of Language. United Kingdom: Cambridge University Press, 1999. 
